\title{
Designing Mobile Applications to support Mental Health Interventions
}

\author{
Mark Matthews $^{(1)}$, Gavin Doherty ${ }^{(1)}$, David Coyle ${ }^{(1)}$ and John Sharry ${ }^{(2)}$ \\ (1) Department of Computer Science, Trinity College Dublin. \\ Phone: +353-1-8961765 Fax: +353-1-6772204 \\ (2) Department of Child and Family Psychiatry, Mater Hospital, Dublin. \\ Phone: +353-1-8961765 Fax: +353-1-8032812 \\ mark.matthews@cs.tcd.ie; gavin.doherty@cs.tcd.ie; david.coyle@cs.tcd.ie; \\ jsharry@mater.ie
}

\begin{abstract}
The advent of mobile technology has brought computing to a wide range of new contexts, some of which are highly sensitive and place new constraints on the designer. In this chapter we discuss issues related to the design and evaluation of mobile software for sensitive situations where access to the end-user is extremely restricted. We focus on the specific example of technological interventions that support adolescents in mental health care settings. We examine the practical and ethical constraints placed on access to end-users and contexts of use, and how this may affect approaches to design and evaluation. General design recommendations for this area are described. We consider approaches to iterative design with mental health care professionals, and how research on technological and therapeutic aspects may proceed in tandem. We identify methods that can be used when conducting evaluation in these limited situations and describe a methodology for maximising the value of such evaluation. By way of illustration, we present the design and evaluation of a mobile phone-based "mood diary" application designed for use in clinical situations by adolescents undergoing mental health interventions.
\end{abstract}

\section{INTRODUCTION}

There are a variety of well established methods for the design and evaluation of user interfaces. Recent developments in mobile technology have brought technology into a variety of novel contexts. Because of this, there has been a focus on ethnographic and context-based approaches to requirements gathering and design. While there are new challenges posed by these contextual factors, by and large designers can draw on adapted versions of tried and tested methods for requirements elicitation, design, usability improvement and evaluation. Hence questionnaires and user interviews, paper prototyping, user observation, think aloud protocols and so on, can be applied in the design and development of new mobile systems.

In some situations, established methods are not viable because of the sensitivity of the environment or the end-users. Sensitive situations, as discussed in this chapter, are situations where poor quality solutions may have a highly negative impact and where

Preprint of paper published as: M.Matthews, G. Doherty, D. Coyle and J. Sharry Designing mobile applications to support mental health interventions, in Handbook of Research on User Interface Design and Evaluation for Mobile Technology, Lumsden Jo (Ed.), IGI, Information Science Reference, pp.635 - 656, 2008. 
the introduction of not just the technology but also the designer could be detrimental to the environment which is the target of the technological intervention. As a result of this sensitivity, one could have a system under development where there is no direct access to end-users. For such situations, it is necessary to identify new methods to address the problems of design and evaluation in these circumstances. This chapter's main focus is on identifying methods for design and evaluation in sensitive situations where access to the end-user is extremely restricted. To achieve this, the chapter concentrates on one particular situation - the use of mobile software in talk-based mental health care with adolescents. An ancillary aim is to give sufficient background detail to help readers understand the significant constraints present in this area. Many of the issues raised are applicable to a wider range of sensitive situations.

This background section begins by briefly describing why adolescent mental health is a particularly sensitive situation and discussing why mobile software has particular promise in this environment. It continues with a description of the ethical constraints the designer needs to consider. We then discuss which traditional usability evaluation methods are applicable in this situation, and discuss related research in this and similar domains. Finally we present a characterization of the end-users; both the adolescent clients and the therapists. The chapter then moves on to present a range of design and evaluation recommendations. In particular, we present a multi-stage methodology for developing mobile software in this sensitive situation and end by describing a case study which demonstrates the benefits of this process.

\section{Mental Health: a Sensitive Situation}

Mental health is one of the most pressing concerns for public healthcare systems worldwide. A large scale international study has identified mental illnesses as the second leading cause of disability and premature mortality in the developed world (Murray \& Lopez, 1996). The past fifty years have witnessed major advances in the treatment of mental illnesses. One significant report concluded that the efficacy of mental health treatments is well documented and that a range of effective treatments exist for most mental disorders (Surgeon General, 1999). Treatment generally takes the form of talking, listening and learning, physical treatment (drugs, ECT, biomedical) and/or social interventions. This chapter focuses on listening-and-talking based intervention methods. Many studies have concluded that talk-based methods are equally as effective as drug based interventions in the treatment of many disorders (Surgeon General, 1999).

When designing technology for mental health care (MHC) environments, researchers have to carefully consider and characterize both the adolescent client and the therapist. Both are sensitive to the introduction of new technologies in a therapeutic setting. Adolescence is the transitional period between childhood and adulthood, and is a time when professional support for dealing with mental illness is in greatest need. However, most adolescents with mental health problems do not receive professional help and those who do can find it difficult to engage with the available services (Offer, Howard, Schonert, \& Ostrov, 1991). Therapy is typically something imposed upon them and because of this they are often unwilling to accept it. Difficulties in engaging adolescents can also arise due to the serious nature that therapy is seen to have in society and the stigma that is often attached to it (BMA, 2006). Exploring new ways to engage adolescents in professional therapeutic services is one of the most important and difficult challenges in mental health. By engagement, we mean helping adolescents develop an active interest and confidence in the outcomes of therapy. 
Sensitivity is required when dealing with therapists who may view technological solutions with suspicion. In medical healthcare, professionals are often uncomfortable to find themselves in the role of computer novice (Gosbee \& Ritchie, 1997). For therapists, this may be the case to an even greater degree as technology is rarely used in day-to-day work or in therapist training. As a result, they may feel the introduction of technology might undermine their authority. Any technology that is introduced needs to compliment a therapist's current practice, methods and not demand too much time in an already busy schedule. Small innovations are required to develop confidence in the possible benefits technology can offer.

\section{Why Mobile Phones?}

Client self-efficacy and the degree to which clients engage with their treatment has been identified as one of the most significant factors in achieving positive therapeutic outcomes (Assay \& Lambert, 1999). Alongside engagement during clinical sessions, a key aim in improving client self-efficacy is to increase the degree to which clients engage in prescribed therapeutic activities between therapeutic sessions. Within public health care systems it is often the case that clients will see therapists at most once a week and sometimes will only attend once a month over the course of treatment. For this reason, it is particularly important to engage clients with the therapeutic process between sessions. Existing (e.g. paper-based) approaches have achieved limited success in improving client engagement between therapeutic sessions. Identified problems with materials currently used include lack of client engagement, stigma associated with therapeutic activities and inconvenience.

The mobile phone is a promising means for achieving greater client engagement between therapeutic sessions. The mobile phone is the most personal communication device with widespread ownership, particularly among adolescents. A recent UK survey indicated that $90 \%$ of 12 year olds owned a mobile phone and sent three times as many text messages as their parents (BBC, 2006). It is an interactive and adolescent-centred technology. Its strengths include ubiquitous and continuous access, familiarity and ease-of-use. It has the potential to make therapeutic activities more convenient, less stigmatic and more engaging.

Adolescents are already familiar with mobile phones and how they function: they use them daily to communicate with peers, play games, and send textual and multimedia content. A high proportion of adolescents are familiar with textual input, using it every time they send a text message. They may find entering text on their phones significantly more appealing and comfortable than using pen and paper, which may have negative connotations. This is very important for clients with literacy difficulties. The phone can simplify the transfer of any client content to a secure server, alleviating the client of the responsibility to do so.

\section{SUPPORTING MENTAL HEALTH INTERVENTIONS}

To provide context for the case study and shed light on the issues involved, we briefly introduce the most important issues to consider when introducing technology in this area. For an extensive review on the use of computers in mental health interventions see (Coyle, Doherty, Matthews, \& Sharry, 2007).

Most adults are comfortable with face-to-face dialogue. This is often not the case with adolescents. Children struggle to express themselves with words alone and much research has been conducted into ways of engaging children using indirect channels 
and play. Adolescents can be resistant to these methods; they like to be treated as adults and will not engage if they perceive they are being treated as a child. Equally, many teenagers are private and self-conscious and often react with confrontation or not at all to direct dialogue with a therapist. Given the general enthusiasm of many adolescents for technology, an opportunity exists to use it to assist with adolescent interventions. Technology may provide a channel through which indirect communication between the therapist and client can proceed in a less confrontational fashion. For example, one study on using a computer game in a therapeutic session, reported that clients found it easier to talk while looking at the screen. (Coyle et al., 2005). It also found that the introduction of the game had an empowering effect on the adolescent clients. They were more comfortable with the technology than many of the therapists.

Previous research outlines the central importance of client factors (client and environment strengths, client resources) and the quality of therapeutic alliance (i.e. the relationship between therapist and client) in affecting positive change through psychotherapy (Assay \& Lambert, 1999). Therapeutic outcomes are more likely to succeed if the therapist engages in a client-centred manner. An effective therapeutic process should actively engage adolescent participation and involve their interests, strengths and ideas. Four core values have been identified as fundamental to establishing a beneficial working relationship: respect, empathy, genuineness and client empowerment (Egan, 2002).

The aims of technological interventions within this area can include acting as an icebreaker, providing material to talk about during the session, re-enforcing coping strategies introduced during therapy, reducing the potentially confrontational and stressful dynamic of a client facing a therapist, and providing peer content so that the client does not feel that they are alone in experiencing difficulties. It is worth noting that a technology intervention need not necessarily have a long term effect. Even an intervention which achieves greater engagement through a "novelty" effect may contribute to the therapeutic process by building a therapeutic relationship or acting as an ice-breaker.

\section{Design under Ethical and User Access Constraints}

Research into the use of technology in talk-based interventions for adolescents must adhere to the strict ethical guidelines of the domain. The sensitivity of this situation is further enhanced by the social stigma often associated with mental illness. Many activities are sensitive and even the most unobtrusive methods of observation by a third party could affect trust and the efficacy of the intervention. All therapists are required to obey the Hippocratic Oath - often summarised by the simple principle 'first do no harm'. This means that above all, every possible precaution must be taken to ensure that interventions do not have harmful effects on the client. Research into the use of technology must adhere to this strict ethical requirement. Roberts and Dyer have produced a guide to MHC ethics and provide solutions to ethical problems (Roberts \& Dyer, 2004). HCI researchers should be aware of these constraints, and with the protocols for evaluation.

Ethical requirements place strict limitations on access to MHC settings. It is unlikely that many HCI researchers will have the required qualifications that would allow direct access to this sensitive setting or for contact with people suffering mental health disorders. It is therefore necessary to find solutions for design and evaluation that take into account these access constraints. Access to therapists is not restricted and they 
can act as proxies in clinical evaluation. An important element of any approach is the ability to maximize the evaluation that could be conducted independently of clinical settings.

There are practical implications to these constraints. To evaluate software in a mental health environment, a detailed proposal has to be submitted to an ethics board. This typically takes at least a month and possibly longer should the board require changes to any part of the study. Furthermore if any substantial changes are made to the software then the ethics review procedure must be performed again. This has a significant impact on the duration of evaluations and reduces the practicality of iterative design with end-users. All software must be thoroughly evaluated, before it can be made generally available. Ethical restrictions rule out user-assisted design and participatory design with adolescent end-users, and also mean less experimentation is possible.

\section{Designing for Adolescents}

The initial problem facing researchers when designing for adolescents in MHC settings is how to characterize them. Since access is extremely limited, we must rely on discussions with therapists and analysis of the research literature.

Adolescents attending therapists suffer from a range of problems including emotional disorders (e.g. anxieties, depression, and phobias), eating disorders, conduct disorders and self-harm. They are a heterogeneous group and have varying backgrounds, motivations, abilities and interests. Socio-economic factors play a major part in the prevalence of mental health problems. A recent survey found that young people living in rented accommodation are twice as likely to suffer from a mental health problem (Office for National Statistics, 2005). Poor opportunities in education and employment are also considered risk factors. Adolescents with learning disabilities are more at risk of developing mental health problems (Royal College of Psychiatrists, 2004). There is a strong sense of stigma surrounding mental illness. Research has identified it as a major concern of adolescents attending therapy (Office of the Deputy Prime Minister, 2004). A particular difficulty is that young people tend to have more discriminatory attitudes to mental health problems than adults.

A recent study has reported that in MHC settings, adolescents need to feel respected, listened to and not judged by health professionals (BMA 2006). They strongly value confidentiality and consistency of care is important. It was found to be essential that the clients do not have to repeat their story to different therapists. It is clear that in respect to these issues, technology can play a valuable role.

\section{Designing for Therapists}

Many therapists have pressurized work schedules and it is important that technologies do not add to this pressure by placing extra demands on their time. An informal survey undertaken by the authors revealed that while many therapists are familiar with Microsoft Office, email and internet software, few are likely to have had specific training or experience in the use of technology in client contact situations. Although computer-based training has many documented benefits and has become increasingly popular in other health science and educational programs, it is still largely unused in therapist training. These factors have a knock-on effect on the use of computers in clinical practice. When questioned about increased use of technology, many therapists express concerns over the need for additional training. They are concerned that existing skills may become obsolete (Caspar, 2004). At this early stage in the 
development of technology for therapeutic interventions it is desirable that systems should be designed to take advantage of the existing skills of therapists and integrate with current working methods.

Many therapists cite fears over the security of sensitive information as one of their primary reasons for scepticism of the benefits of technology (pers. comm.). While many methods have evolved for ensuring the actual security of electronic data, perhaps more significant is the perceived security of this information by both therapists and their clients. In peer support and collaborative group systems it is also essential that the accuracy of information and the trust of individuals be maintained and represented. Maximizing this sense of security and trust is thus an important topic for further HCI research in this area.

It is important that the introduction of technology involves protocols for implementation and use. Such protocols can prevent misunderstandings and ease concerns related to the use of the technology. A particular concern for therapists is that the introduction of a new technology brings greater responsibility and more opportunities for client-therapist contact. For example, some therapists were concerned that the introduction of mobile technology may add to their workload and responsibility by introducing a constant line of communication between them and a client which they would feel obliged to monitor. Designers have to take such concerns into consideration.

\section{Mobile Software to Support Psychotherapy}

Adolescents spend less than one hour a week on average in a therapeutic session. Mobile technology holds promise for engaging adolescents when they are away from the session. It is a portal into adolescents' personal space and can offer them persistent and continual access to therapeutic materials and activities outside of the therapy session. It affords the user a level of privacy and security unattainable with other technologies. It is a simple matter to provide a mechanism to secure personal and sensitive information. Using a mobile phone in public is a common and accepted activity. The privacy and security provided by the mobile phone may contribute to greater candour. Research has reported that SMS users are more likely to be intimate and revealing in text messages than in face-to-face conversations (Schaefer \& Reid, 2001).

As a networked and an interactive device, the mobile phone can initiate therapeutic activities. Clients can be sent reminders via their phones to perform a particular task. Mobile content can be automatically uploaded to a secure location which greatly reduces the possibility of the loss of information and subsequent loss of trust in the software. As many adolescents carry their phone almost everywhere they go and do not have to make an extra effort to remember their therapeutic materials. The use of the mobile phone may help to overcome some of the barriers to treatment by providing access to therapeutic materials and activities regardless of location and clinic opening hours. Introducing a device which has many positive connotations for adolescents may also help to offset some of the stigma associated with attending therapeutic sessions. Another advantage in this context is that it gives access to periods where the client is unoccupied or bored, when they might willingly engage in therapeutic activities.

A successful implementation using mobile technology may support the therapeutic process by: (1) creating content for use in a session, (2) engaging adolescents in 
beneficial therapeutic activities, (3) encouraging them to spend more time thinking about therapeutic matters and (4) making therapeutic material more convenient and accessible.

There has been considerable research into using mobile technologies in education (Naismith, Lonsdale, Vavoula, \& Sharples, 2004; Roschelle, Sharples, \& Chan, 2005; Savill-Smith, 2005). Some of this research can be relevant to mental health care as it deals with children in similar technological environments where pen and paper activities are still dominant. It is an area that requires some sensitivity, albeit significantly less than clinical situations. One project used mobile phones with schoolchildren as a bridge to a web portal (Vavoula, Sharples, Rudman, Lonsdale, \& Meek, 2006). The mobile phone was used for data collection on the move around an art gallery. The content collected was later viewed and organized through a web portal, which allowed for more complicated interaction. This project found that the mobile phone was less fragile and more engaging than the paper alternatives. This project used a process where evaluation and development took place at the same time (Vavoula, Meek, Sharples, Lonsdale, \& Rudman, 2006). Outcomes from one stage of the development informed the next stage.

Mobile software has been demonstrated to hold much promise in general health care, potentially supporting more attentive care at a reduced cost and with more comfort to the patient. Text messaging (SMS) has been used to generate daily medication reminders (Neville, Greene, McLeod, Tracy, \& Surie, 2002). Systems for monitoring dialysis (Nakamoto et al., 2003) and asthma (Sanderson, Andrews, Corry, \& Lapsley, 2003) have been developed. One project developed a rapid application development methodology for producing personalized educational software for breast cancer patients (Wood, Keen, Basu, \& Robertshaw, 2003). However, there is a significant difference between producing general healthcare applications and producing software for users with psychological problems, who may be considered fragile. There is the added complication of stigma and the potential embarrassment associated with mental illness. This is magnified when end-users are adolescents. Nevertheless, examining approaches and methodologies from similar areas can have benefits for research in this area.

Research into using the mobile phone as a therapeutic support device is at an initial stage. Few, if any, studies have looked at HCI issues but have focussed generally on therapeutic evaluation. One study using SMS messages in the aftercare of bulimia nervosa patients outlined the following benefits of mobile technology (Bauer, Percevic, Okon, Meerman, \& Kordy, 2003): (1) access is independent of space and time, (2) there is little temporal or monetary cost, (3) it is an interactive medium, (4) patients are encouraged to express themselves briefly and concisely, (5) it allows patients to stay in contact with therapy and (6) it can help to bridge the gap between inpatient and outpatient treatment.

Using the mobile phone may encourage adolescents to consider therapeutic matters. One study, which used PDAs as an extension to therapy, reported that signals from the device reminded clients to think about things like eating behaviour and mood (Norton, Wonderlich, Myers, Mitchell, \& Crosby, 2003).

The mobile phone provides an increasingly powerful and ubiquitous platform with the potential to support a variety of applications. The types of therapeutic activity that are most suited to mobile phones have yet to be established. Functions that are familiar to adolescents (e.g. entering text, taking photos) may have powerful applications in this 
area, including content collection, story-telling and psycho-education. There is an opportunity to build on developments with mobile phones in less restricted areas such as education, while considering the particular characteristics of the mental health context which bring with it a different set of design concerns.

\section{RECOMMENDATIONS}

The following sections look at the design and evaluation of mobile software for adolescents and therapists involved in professional mental health services. Traditional methods for design and evaluation must be adapted. We present a number of recommendations based on our experience working in this area. Careful design is essential to ensure that technology acts as an effective support to the therapeutic process and not a barrier.

\section{Interaction Design Methodologies}

Existing approaches to interaction design and usability improvement involve a number of stages. These range from high level requirements gathering and analysis, to low level, detailed design. A range of techniques is used to improve usability and ensure compatibility with user needs. These techniques involve end-users - either directly (as with participatory design), or as a source of information for analysis (as with ethnography and contextual analysis), or to validate and explore designs (often low-fidelity and 'rapid' prototypes). Even with analytic approaches (such as task and activity analysis), end-user validation of models is seen as an important aspect.

In recent years, there has been an emphasis on studying applications in the context where they will be used (Suchman 1987). Researchers and designers looking at interaction in a mobile context may need to employ a combination of new and established data gathering and evaluation methods.

Only relatively recently have children (including adolescents) been studied as a distinct group in HCI (Brukman \& Bandlow, 2002). Druin presented a framework describing the roles that children can play in the evaluation of technology and urges active participation from children as design partners (Druin, 2002). Markopoulus et al. have written on the assessment of evaluation methods for children (Markopoulos \& Bekker, 2003). There are detailed guidelines for HCI researchers working with children (Barendregt \& Bekker, 2005; Hanna, Risden, \& Alexander, 1997; Hanna, Risden, Czerwinski, \& Alexander, 1999). These approaches assume some degree of contact with end-users, and hence in this domain are subject to the same access difficulties as standard HCI techniques.

Given the lack of direct access to end-users and the need to test by proxy through therapists, it is unavoidable that the time taken to feed results back from end-users is greatly increased. Coupled with the constraints on information gathering, this effectively means that we cannot recommend applying standard approaches to usercentred design without modification. As there is an imbalance in access to the two user groups, the obvious option is to rely heavily on therapist evaluation and opinions on designs. While it is appropriate to see the goal of the designer in terms of providing technology support to therapists, full exploitation of the possibilities of technology within such areas can only come from understanding the relationship and interaction between both types of end-users and technology. 


\section{Data Collection}

In the limited settings of mental health interventions it is important to gather as much information from evaluations as possible and make the most of the available evaluation methods. Furthermore, because new technology must be evaluated from both a therapeutic and a HCI perspective there is the risk of overburdening the therapist and client.

It is extremely difficult to obtain fine grained contextual data on the use of mobile applications by adolescent clients. When software is evaluated in a therapeutic session the therapist is a witness to how the technology is used by the client. With mobile software it is difficult to know what context it is being used in. Implementing application logging within mobile applications goes some way to providing interaction information. Available measures include time stamping interactions, analyzing time spent on task and examining which functions of the software were used and with what frequency. While this process is potentially invasive to the privacy of the adolescent client, the benefits to be gained justify the measure, provided that there are strict protocols for dealing with this information. This information can provide insights into what areas of the software adolescents are spending most time on and any difficulties they may be experiencing. Depending on the purpose of the software, further measures may be possible. For example, if it is primarily designed to gather client content, then appraising the quality and frequency of this content can go some way to evaluating its effectiveness.

In clinical trials, therapists participating in evaluations must agree to act as proxies. They are then responsible for administering pre- and post-questionnaires to their clients. As time is limited, it is not reasonable to expect clients to spend a long time in a session filling out questionnaires. For this reason they have to be succinct and clear. They should allow clients the opportunity to openly express their opinion on the software, as well as gathering information about use of the software. Protocols for data collection should provide clients with privacy and anonymity when completing questionnaires. Therapists must give clients space to express their opinion. In order to allay client concerns that their therapist might read their questionnaires, it is advisable to seal them in envelopes once completed. Due to the constraints of the setting it is generally not possible to have therapists administer interviews to their clients.

The HCI researcher can ask a little more of participating therapists, who are often enthusiastic, and keen to add to their repertoire of therapeutic tools. Questionnaires and interviews with therapists before and after testing are permissible. The focus is on how the technology affected their usual practice and their impression of how the adolescent perceived the technological intervention. In many cases, therapists may have a client attend over a period of months before, during and after a technological intervention. This gives them a good perspective to assess the affect the technology had on the client and the intervention.

The evaluation of mobile software for clinical settings is a long process that begins with sending detailed proposals to an ethics board and involves long delays while therapists wait for the appropriate time to introduce the mobile application into an intervention. Testing can overrun, due to clients' poor attendance and other factors. These delays mean that clinical evaluations cannot reasonably form the basis for an iterative design process. 


\section{Information Gathering Methods}

The advent of mobile computing has had significant impact on the HCI field, underlining the importance of context. Significant challenges have arisen regarding the conduct of usability studies for mobile systems, particularly concerning the gathering and analysis of data, and the difficulty of isolating contextual factors. Innovative solutions are sometimes required - one non-mental health study examined the use of mobile phones by 16-22 year olds (Carroll, Howard, Peck, \& Murphy, 2002). It made use of several evaluation methods including scrapbooks with disposable cameras, online diaries and focus groups. However, in a MHC setting the restrictions on access make such rich information gathering difficult - data collection in clinical settings is severely limited and unsatisfactory for the purposes of usability improvement. This affects both requirements analysis and usability improvement activities.

Below is a consideration of some common information gathering methods used in requirements analysis and usability improvement, and the possible use of these methods in sensitive settings such as adolescent mental health care.

Interviews and discussion groups - Discussion groups have been used in nonmental health studies with adolescents to good effect, see for example (Grinter \& Eldridge, 2001). However due to the ethical constraints, interviews and discussion groups between HCI researchers and adolescents in therapy are effectively impossible. By contrast, interviews with therapists are a valuable source of information.

Think-aloud evaluation - Think-alouds have been used in non-mental health studies with adolescents to examine their interaction with cd-rom encyclopaedias (Branch, 2001). However they can be problematic with mobile systems, although not impossible with appropriate equipment. For sensitive situations however, they are subject to the same ethical constraints which curtail the use of video and audio logging.

User notebooks - User notebooks are often used for longitudinal studies and have the advantage of being applicable to mobile systems, particularly where interaction with a system is relatively sparse and spread over a long period. There are difficulties recording entries in notebooks when the users are on the move (Grinter \& Eldridge, 2001). For many sensitive situations, this approach is unlikely to work because of privacy concerns (in some cases related to stigma), or because the end-users are unlikely to find completing the notebook entries to be an engaging activity (as with adolescents). One non-mental health HCI study, taking this into consideration, implemented a voice mail diary technique for participants in a mobile technology study (Leysia \& Marilyn, 2002). This may be worth exploring, although it is likely that any HCI evaluation technique which requires adolescent clients to focus more on usability evaluation than therapeutic engagement will not be acceptable. As it stands, it is extremely difficult to engage adolescents in therapeutic activities.

Questionnaires - These can be administered by proxy without loss of confidence in the accuracy of the data. Because of the controlled nature of use, the completion rates can be very high.

Application logging - Software is available to record fine grained data of users' interactions; for example, cursor movement on desktop computers. The mobile 
phone, with its limited power and storage capacity, does not support intensive operations such as screen capture. More modest logging is possible, such as navigation choices and time spent on task. This can be a useful means of gathering data once client and therapist approval has been established. Appropriate permissions and awareness of this data collection must be part of the protocol.

User observation - User observation in context often involves either following users and observing their mobile use from afar or end-users wearing special video recording equipment. Both methods are inappropriate in adolescent mental health settings. It may be argued that observation of therapy sessions using video recordings could be used as an alternative to direct contact. However, given the ethical constraints and privacy demanded of therapeutic interventions, even limited second hand observations are likely to prove ethically sensitive, time consuming to organise and prohibitive to regular use. Even in the training of therapists there is a reliance on role-play rather than direct contact. While video of real sessions is used in training of therapists, it is not straightforward to receive clearance to make such recordings. Moreover, it is not generally possible for mobile users. A further difference is that video recordings made for training purposes can be reused many times over a period of years. This justifies the effort to overcome the ethical issues involved and is a consideration which does not apply to usability improvement.

\section{Mobile Design Recommendations}

The following guidelines are intended as high-level recommendations for the design of mobile software in mental health situations with adolescents:

(1) Ethical requirements: Research into the use of mobile technology in MHC settings must adhere to strict ethical requirements. To help in meeting these requirements we suggest that new systems be (1) based on accepted theoretical models of MHC, (2) designed in full collaboration with therapists, (3) designed to integrate with existing working methods and (4) used by clients under the guidance of a professional therapist. Prior to being used in clinical settings it is essential that (1) the reliability and usability of any new system is thoroughly verified, (2) the therapeutic validity of systems is evaluated by therapists and (3) ethical clearance must be agreed for all proposed studies. The process of validating systems and gaining ethical clearance is a time consuming process which must be completed prior to each stage of the clinical evaluation of a system.

Protocols should be provided for adolescents, therapists and parents/guardians regarding use of the software. It is particularly important to provide reassurance to the adolescent that they have full control of access to their personal information.

(2) Design for privacy and engagement: Privacy and security are deeply intertwined for end-users. It is a common sight to see people interacting with their phone in public, and so it is straightforward for an adolescent to avoid attracting attention when using mobile software. The small display screen and one-handed text entry provide increased privacy. Mobile software should be easily locked with a pin so the adolescent alone has access to their information, providing a sense of security. Should the adolescent's phone be lost or even taken off them by a peer, there is no fear that their data can be accessed. It is also vital that the software remains sensitive to the 
possible situations it might be used in. Above all it should avoid attracting attention since an adolescent may use it in public situations. For example, an application should avoid playing any audio files without explicit consent from the end-user.

Software design should seek to engage disinterested adolescent clients in particular tasks or subjects. In sensitive situations it is often inappropriate to enforce use of mobile software. It can be made visually attractive in ways that current therapeutic materials do not afford. Use of animation, colour and the interaction possibilities the phone affords, may make therapeutic activities more appealing. It may also be possible to give rewards such as unlocking simple games or some form of feedback based on a client's use of the software. Responses other than default values should be encouraged. This could entail making the interaction sufficiently engaging or enjoyable that it draws the user in.

(3) Ease of use: Mobile software should require as little training as possible for both clients and therapists. It should also require very little technical support. Clinical settings allow no direct contact between HCI researchers and end-users. It is generally only possible to give automated support to adolescent clients, like for example the automated sending of forgotten passwords. While it is possible to give more technical support to therapists - it should be a last resort. Therapists are unlikely to use software that requires significant training at the outset or relies on frequent support.

Because new technological interventions are almost always introduced to clients by therapists, it is essential that they have a clear understanding of them. We recommend presenting any software well in advance to therapists in order to give them time to become comfortable with it. A short introduction session can help to demonstrate the purpose of the software. It should also involve giving detailed suggestions on how therapists might introduce it to a client. Finally, it is invaluable to get therapists trying the software themselves while there is support on hand. Role play is extremely useful. Therapists can take turns playing the role of therapist and playing mock-clients. This helps them practice how they might introduce the software to a client and also brings up questions and queries that can be dealt with on the spot. Above all it helps them gain confidence in the technology. Many therapists are particularly comfortable with role-playing as it often is part of their training.

(4) Personal mobile phones. Many mobile HCI studies evaluate mobile software designed for PDAs or high-end phones which are then given to end-users. This is justifiable for many research projects and numerous situations. In this area, we believe it is vital to use clients' own mobile phones. They are a technology in widespread use and represent a practical solution in terms of cost and accessibility. PDAs, while becoming less expensive every year, still carry a significant hardware cost. They require upkeep and technical training and may introduce a novel element into testing which would be difficult to evaluate. Many adolescents will not have used one before and may attract unwanted attention while using one in public. Adolescents already carry their phone wherever they go and are familiar with how it works.

The decision to use the client's own phone has implications for design and implementation. To achieve necessary coverage, software has to function on a wide range of models, including older models. Designing for such a broad base brings significant restrictions. Almost every device uses different hardware and a different user interface. It is a challenge to design and develop software for all devices in a particular market. The mobile version of java (JavaME) enables the researcher to 
design mobile software that will function and appear as a native application on each different handset. This has the advantage of building on the user's existing knowledge of their device.

(5) Access: Practical, time and ethical constraints require that any mobile software be accessible to as wide a group as possible. Software should therefore function on a wide variety of handsets, to avoid the potential stigma of it not working on certain older models and to speed up evaluation. Although increasing access requires more application development time, it should result in quicker evaluation because the software will be available to a greater number of clients. This means that design is restricted to the limitations of the least advanced phone in the market, unless of course researchers are prepared to develop several versions of the software for different models.

(6) Standard UI and design: Mobile software should use the standard user interface and interaction style of the user's native phone wherever possible, although a balance has to be struck between this and providing an attractive and engaging interface. Design - graphical and otherwise - should strike a familiar note with an emphasis on privacy, security and transparency. Palmblad and Tiplady have made several recommendations for the design of mobile user interfaces for electronic patient reported outcomes (ePRO) which are informative (Palmblad \& Tiplady, 2004). While their suggestions are directed toward general patient use, particularly on PDAs, many are relevant here. For example, they argue that questions presented to the user should require an active response and that all attempts should be made to keep content on one mobile screen to require no scrolling. For our purposes this entails designing for the smallest screen on the market. They also make recommendations regarding text size, colour and screen layout. It is important to remember that many end-users may have learning difficulties, and so in many cases text should be kept to a minimum and be as informal as possible.

(7) Error handling: As with any well designed application, program errors should be explained practically to the user and without use of technical jargon. Software should handle the burden of any failure, making any necessary operations in the background without requiring any further action from the end-user or any further notification. Information loss must be avoided at all costs. Adolescents resent having to repeat their stories to therapists and are likely to resent having to re-record any content.

\section{Adaptable Systems}

As highlighted earlier, client factors and the ability of a therapist to work in a clientcentred way are critical factors in the success of mental health interventions. Professionals working in public health care systems are faced with the need to work with a broad range of clients, from a wide range of socio-cultural backgrounds and experiencing a broad range of disorders. Working in such situations requires therapists to be able to adapt their approaches to suit the needs of a given client. Socio-cultural issues are one of the major factors in the development of mental health disorders and are a key factor which must be considered in treatment. Some of the specific socio-cultural issues which affect mental health care include: gender, age, social class, sexual orientation, religious or secular assumptions and race, culture and ethnicity. Systems designed for mental health interventions should ideally reflect this need for adaptation to the needs of clients. Further forms of adaptation are also desirable. For example, teams of therapists working in public health care settings 
often have different theoretical backgrounds and many adopt a variety of eclectic approaches to working with clients.

For technologies to be of practical use in a broad range of MHC interventions, they should ideally be adaptable to (1) a broad range of theoretical models, (2) a broad range of mental health disorders, (3) the differing needs of various demographic groups, and (4) the specific needs of individual clients.

The authors are investigating a form of adaptation which allows therapists to adapt the therapeutic content delivered by systems. For example, some mobile software may include a reminder element where clients receive text message reminders. Different clients may like to receive these at different intervals or at different times. This needs to be easily modifiable. More importantly, the content of these reminder messages needs to be easily adaptable by therapists to the specific needs and motivations of each client. Just as a therapist needs to choose the suitable therapeutic tool for a particular client, they should be easily able to tailor the mobile application.

Adaptable systems offer therapists the flexibility to adjust systems to meet the needs of given clients or client groups. While HCI researchers focus on the design and development of frameworks for delivering therapeutic content, MHC researchers can adapt these systems for use in various interventions and evaluate their therapeutic benefits. Whilst it is desirable that a large number of therapists are comfortable using any system, it is not necessary that all therapists be able to make large scale modifications in the use or therapeutic content of a system. A possible model of use for such a system is:

Small groups of expert MHC researchers and therapists develop and adapt content for use within their own areas of expertise e.g. targeting specific disorders and implementing specific intervention models.

Larger groups of therapists use these implementations with clients and have the option of making small scale adaptations to suit the needs of specific demographic groups or clients.

By enabling therapists to play an active role in building and tailoring the content delivered by computerized systems, adaptable technologies provide a sustainable approach to the development of client and therapist-centred technologies for mental health interventions.

\section{Therapists and Designers Work in Parallel}

While collaborative design, involving domain experts, is desirable in most design spaces it has a particular significance in MHC. Difficulties caused by lack of access and ethical constraints are further exacerbated by the fact that few therapists currently have the experience required to design or develop new technologies or to rigorously evaluate them to the standards required for successful introduction to clinical settings. In order to overcome these limitations, the design and evaluation of mobile applications requires the close collaboration of HCI researchers and therapists.

At the outset of the design process there needs to be a cross-pollination of ideas between the two and a sharing of knowledge from each expert's domain. The HCI researcher needs to appreciate the sensitivity of the situation and to be able to characterize potential adolescent clients through conversation with the therapist and to understand a therapist's motivations and typical working practice. The therapist needs to be made aware of the possibilities and benefits afforded by the technology, the 
things that might not go to plan and how to present the technology to the client. Therapists must develop protocols which include recommendations for use of technology in practice. Questions of when it is appropriate to use a piece of software, how long it should be used for, and how it should be incorporated into the therapeutic process are questions which lie in the domain of mental health research. Hence, a parallel cycle of development of protocols can be carried out by mental health therapists, while still feeding back information of relevance to the design and usability of the application.

The HCI researcher and therapist both contribute to identifying needs or areas where technology can add to the situation and to developing and appraising the initial application design. Characterization of end-users can proceed through collaboration with therapists and detailed analysis of the relevant literature. But it is clear that some questions only answerable by end-users. Both parties are interested in ensuring the best outcome for the client, but where the therapist is focused on the overall effectiveness of the intervention, the HCI researcher is concerned with designing the most effective software.

\section{Multi-stage Prototyping}

Ethical and access restrictions make iterative design and user-centred design problematic. In order to overcome these limitations and to maximise evaluation possibilities, we advocate a three stage approach for evaluating mobile software (see Figure 1). In the first two stages, these groups consist of adolescents from similar backgrounds to the targeted end-users, but who do not have mental health problems. The most detailed HCI evaluation takes place in the early stages of this process while there are fewer limitations to the methodologies available. At each subsequent stage the evaluation and data collection possibilities become restricted as access and ethical limitations increase. The main goal of this section is to describe a process that refines design through the use of adolescent peer groups.

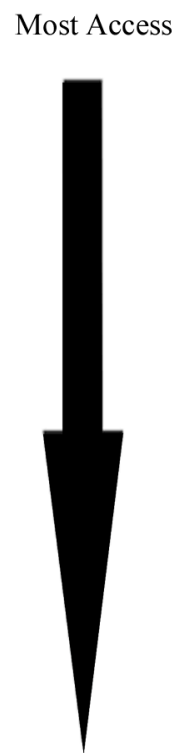

Least Access

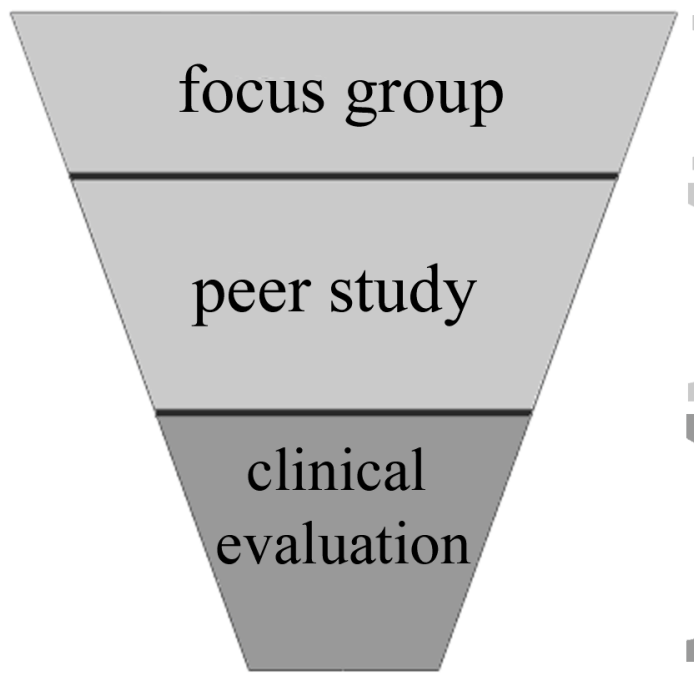

- think aloud evaluation - application logging - questionnaires - face-to-face interviews - direct observation - application logging - content analysis - questionnaires - face-to-face interviews

- application logging - content analysis

- end-user questionnaires - therapist questionnaires - therapist interviews

$=$ non-clinical user group $=$ clinical user group

Figure 1 - Three stage evaluation 


\section{Stage one: Focus Group}

As described earlier, a close working relationship between the HCI researcher and therapist is essential as a foundation to the design process at this stage. At this stage, once a particular design has been identified, several paper prototypes are created. Paper prototypes are particularly useful in providing initial common ground for the two parties, who are at the outset approaching the design from very different perspectives. They help open a dialogue between the therapist and the HCI researcher around different designs, leading to more focused and detailed designs. These designs are initially tested informally with colleagues from HCI and mental health backgrounds. A working prototype is developed once a particular design has been chosen. Next a pilot group of between five and ten adolescents is formed. These adolescents do not have mental health problems but are chosen based on similar socio-economic, educational and geographical backgrounds to the ultimate adolescent end-users. The prototype is loaded onto participants' own handsets and detailed qualitative data can be gathered through face-to-face interaction with researchers. Evaluation at this stage focuses on usability and the overall appeal of the software. Evaluation methods available at this stage include: think aloud evaluation, application logging, questionnaires, interviews and direct observation in a controlled environment.

\section{Stage two: Peer Group User Study}

The data collected from stage one is collated and analyzed. It is used to inform any changes to the original prototype. Substantial design changes can still be made at this point. Once these changes have been implemented, the software is tested on a larger group of adolescent peers (typically twenty to fifty), again from similar backgrounds to the intended end-users. This stage aims to evaluate the design across a wider group of adolescents, with sufficient numbers to obtain statistically significant results.

Schools are the most convenient partners to obtain access to large groups of adolescents. Testing can take place over a number of weeks, depending on the requirements of the evaluation. Whether training or technical support are provided again depends on the mobile software being evaluated and the aim of the study. A short group introduction should be given to participants explaining the purpose of the testing. It should be made clear that it is the software rather than the participant which is being tested. The software is then loaded onto the phones of participating adolescents. Data collection at this stage depends on what exactly is being evaluated and can involve qualitative and quantitative measures. There is a focus on usability across a wide range of users and handsets as well as on content created and emotional engagement with the software. Measures appropriate include: application logging, analysis of any content created if relevant to the mobile software being tested, questionnaires and face-to-face interviews. Access and ethical restrictions are still relatively relaxed at this stage, but the number of participants inhibits extensive qualitative data collection.

\section{Stage three: Clinical End-User Evaluation}

This is the final stage of evaluation. Research must not proceed to this stage unless there is confidence in the outcomes. It begins by analyzing all the data from the previous stage and implementing changes into the final prototype. This stage of testing involves clinical trials with adolescents who are attending therapists. The number of participants will vary according to research resources and therapist and 
client availability. The authors recommend fifteen to twenty as a manageable number. Evaluation proceeds in conjunction with the participating therapists. Available data collection measures include: application logging, content analysis, succinct end-user questionnaires, therapist questionnaires and interviews. Analysis of the content collected is particularly important. Questionnaires are possible at the end of testing, but should be short and need to address some therapeutic elements. Short interviews may be possible via proxy, but will include a therapeutic perspective. More detailed feedback can be collected from the therapist via questionnaire and interviews, covering the impression the technology had on the client.

Once these three stages have been completed it is possible to implement final changes and make the software generally available to therapists. The objective of this design approach is to reduce the burden of evaluating the mobile software for therapists and end-users to a minimum, as well as reducing the time taken to iterate through design approaches with adolescent peers and increase the amount of feedback on the design. What follows is a case study giving a specific example of this process in action. This study serves not to present any particular results but rather as a holistic example of the methodology in practice. It also serves to demonstrate some of the recommendations discussed earlier.

\section{CASE STUDY: 'MOBILE MOOD CHARTING'}

As an illustration of the principles and methodologies discussed, we describe the design and evaluation of one particular mobile application called Mobile Mood Diary. It is a mobile phone version of a paper diary that therapists often give to clients to monitor mood. The main significance of this case study, as presented, is as a demonstration of the multi-stage prototyping process. We will not go into specific analysis of individual evaluation of elements of the software, although we will touch upon them in passing. This section is focussed on discussion of the practicalities of putting this methodology into practice.

\begin{tabular}{|c|c|c|c|c|}
\hline $\begin{array}{l}\text { Date } \\
\text { \&Time }\end{array}$ & $\begin{array}{l}\text { Energy } \\
\text { How much energy do } \\
\text { you have out of ten? } \\
\text { (please put an ' } X \text { ' on } \\
\text { the scale) }\end{array}$ & $\begin{array}{c}\text { Sleep } \\
\text { How many hours } \\
\text { did you sleep last } \\
\text { night? }\end{array}$ & $\begin{array}{c}\text { Mood } \\
\text { How do you feel today } \\
\text { out of ten? } \\
\text { (please put an ' } X \text { ' on the } \\
\text { scale) }\end{array}$ & $\begin{array}{c}\text { Diary - please describe how } \\
\text { you feel today. }\end{array}$ \\
\hline \multirow[t]{2}{*}{$\begin{array}{r}1 / 05 / 06 \\
10.15\end{array}$} & 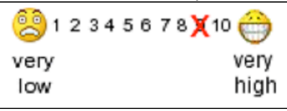 & I slept __ 8 _ hours. & $\begin{array}{r}81234567897 \times \circlearrowleft \\
\begin{array}{c}\text { very } \\
\text { low }\end{array} \\
\text { happy }\end{array}$ & $\begin{array}{l}\text { I feel great today, loads of } \\
\text { energy. }\end{array}$ \\
\hline & $\begin{array}{rr}60 & 2345678910 \bigoplus \\
\text { very } & \text { very } \\
\text { low } & \text { high }\end{array}$ & I slept ___ hours. & $\begin{array}{r}\Leftrightarrow 12345678910 \bigcirc \\
\begin{array}{r}\text { very } \\
\text { low } \\
\text { happy }\end{array}\end{array}$ & \\
\hline
\end{tabular}

Figure 2 - Fragment of paper diary

'Mood monitoring' is an important component of Cognitive Behavioural Therapy (CBT). This involves adolescents recording their mood at regular intervals, to help them to recognize the factors which may be impacting on their mood and how they have managed at times when they have felt depressed. 'Mood monitoring' is traditionally done as a pen and paper exercise which adolescents are asked to complete on a daily basis, using a mood chart supplied by the therapist. Young people are notoriously poor at completing these charts or remembering to bring them to the next session (Feldman Barrett \& Barrett, 2001; Lester, 1999). This case study describes a comparative evaluation of a mobile mood diary and a paper mood diary (see Figure 2). 


\section{Design of the Mobile Chart}

The initial design of the mood diary involved one dedicated HCI researcher and one therapist working together in consultation with several other HCI experts and therapists. The main goal of the software was to enable adolescents to record their mood (as well as energy, hours of sleep and diary recording) in a streamlined process. Recording a mood needed to be a series of clear and familiar actions that could be accomplished quickly by adolescent end-users. The objective was to make the software as intuitive to use as a paper diary.

In accordance with the design recommendation to provide wide access, the software had to work on the majority of adolescents' own phones. From a technical viewpoint, it was important to appraise different solutions to achieving this. The transfer of data is possible via IR, Bluetooth or HTTP; however, the criterion of access ruled the first two out as current phones in the adolescent market did not provide sufficient support. Furthermore, both technologies would have required that the information be transferred during a therapy session, potentially taking up valuable time. It is also possible that there might have been technical hitches which could have undermined the client's confidence in the therapist.

An initial survey of current phones on the market over the past year revealed that there was stronger support for JavaME than other software platforms. Further surveys at the first two stages of evaluation supported this, revealing an even wider support base. This meant that clinical evaluation could proceed with the confidence that the majority of client devices would be supported by the software. JavaME provided a reliable method of providing an interface which behaves and looks like the native user interface of each individual phone, as well as a robust way of reaching a wide number of existing handsets.
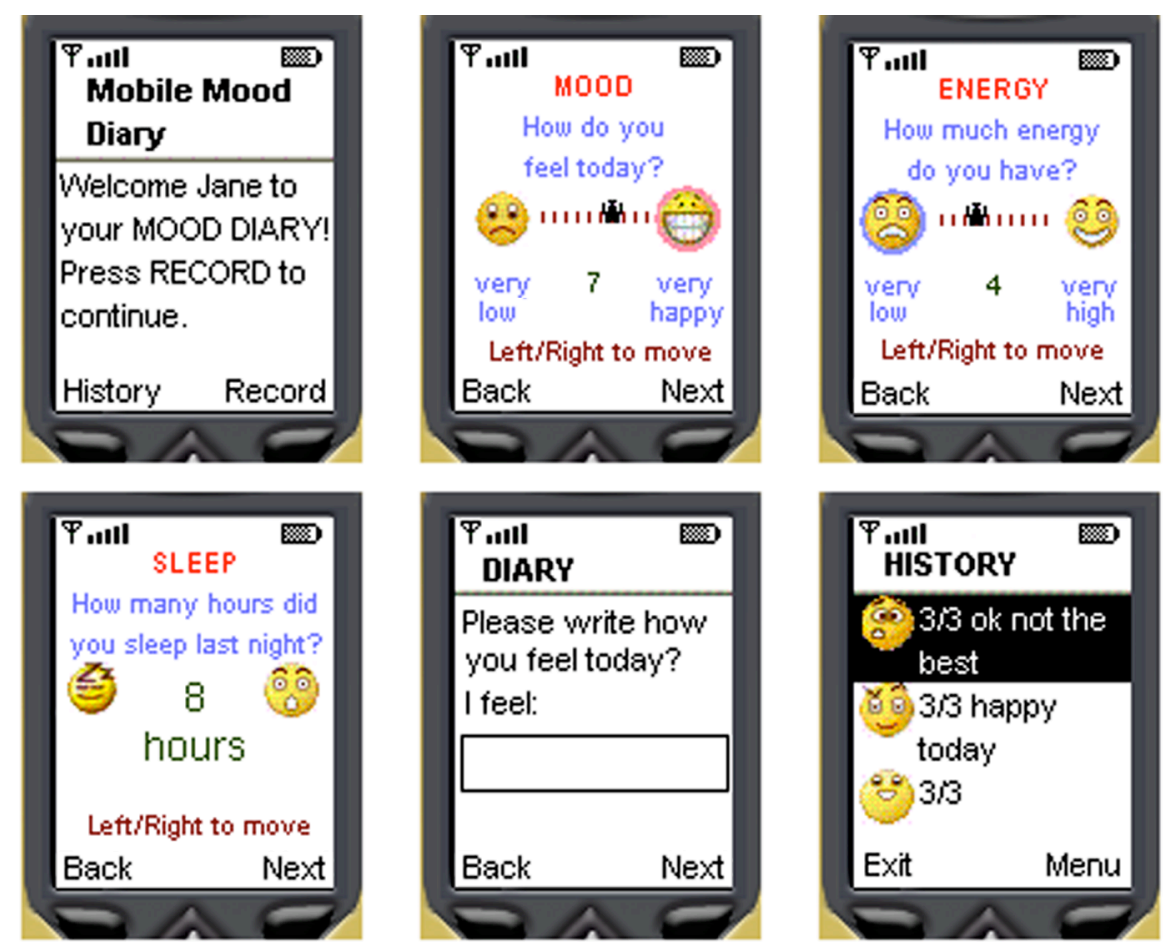

Figure 3 - Screens from final mobile diary design 


\section{Stage one: Focus Group User Study}

The initial prototype of the mobile mood diary included sliding bars for recording mood and energy (both out of 10), as well as hours slept and a space for texting thoughts. A single mobile screen was used for all screen elements. Initial testing took place with six twelve to fourteen year olds in an inner-city youth club and was in line with the wide range of activities carried out there. The application was installed on their phones and they were asked to complete a series of simple tasks, while following the think-aloud protocol. They were then allowed thirty minutes free use of the software and then interviewed for general opinions. They also completed questionnaires.

Significant usability issues with the initial prototype were identified at this stage. Some participants failed to notice some screen elements and became frustrated with the interaction of selecting different screen objects. On some handsets this required vertical scrolling to navigate the content. As a result of the initial evaluation, the prototype was changed to include four distinct pages, one for each of the elements to be recorded (see Figure 3). Other modifications included making the user interface more graphically appealing, replacing the default standard scale with a game metaphor of a moving character along a one to ten scale and streamlining the navigation structure.

The purpose of this stage was to reveal any significant interaction difficulties and get detailed usability feedback from adolescent users. This evaluation stage allowed for hands-on involvement from designers and the opportunity for participants to actively contribute to the design process. By the end of this stage, there was a clear idea of what worked and what needed to be changed.

\section{Stage Two: Peer Study}

Following significant redesign, a second evaluation in three different schools was carried out. A non-clinical sample of seventy self-selecting students took part in the study with school and parental permission. Participants' ages ranged from thirteen to seventeen $(n=73$ mean $=14.87 S D=1.141)$ and were mostly female $(86.3 \%)$. Fifty-two students were given paper-based charts and twenty-one students had the mood diary loaded onto their phones. The purpose of this stage was to evaluate compliance and usability of both the paper and mobile diary. Due to the emphasis on entering real life data (i.e. moods) and the more formal setting, a greater overhead was involved in securing access and parental consent, and care had to be taken regarding privacy. The experiment proposal was reviewed by an ethics board in the same fashion as a clinical evaluation. While a full description of this study is beyond the scope of this chapter, we present the format and main parameters, along with a summary of the results.

Testing took place over a two week period. Both groups were asked to record their mood once a day, although they were not reminded to do so. No incentive was given to either group. Data collection at this stage included data logging, analysis of the recorded content and post-experiment questionnaires. Post-trial face-to-face interviews were also used with both groups to gauge general opinions, overall satisfaction and suggested improvements.

In order to assess whether a paper versus mobile phone-based platform influenced compliance behaviours, participants who completed paper based diaries $(n=16)$ and participants who completed a mobile phone based diary $(n=17)$ were assigned to two groups. Compliance was judged as completing at least one entry per day. Data was 
analysed using an independent samples t-test in order to test for significant differences in the number of mood entries participants completed. Groups were found to differ significantly in their rates of actual compliance $(t=-2.324, p<.027)$, with the mobile phone based diary group (mean $=8.12)$ producing significantly more entries than the paper based diary group (mean $=5.44)$.

The study also provided qualitative data which indicated that the adolescents who were using the mobile phone were happier with the level of privacy the phone provided and found it as easy to use as the paper method and significantly more appealing. Feedback strongly suggested that adolescents valued security and suggested that a security feature (e.g. a pin) be added to the software. Overall it was a successful evaluation of the mobile mood diary, which did not require a technical demonstration or technical support during the testing period. The strength of the results provided ethical justification for proceeding to the third stage of the evaluation, firstly as there clearly seemed to be a benefit associated with the software, and secondly that no technical or major usability issues arose over the two week period of each evaluation.

\section{Stage Three: Clinical End-User Evaluation}

By the clinical stage, the software had been evaluated with a large number of adolescents. The analysis of feedback received from the school pilot study was used to make modifications to the mobile software. Additions suggested from stage two, like a security pin, were added. Beyond the software itself, it became clear that it is important to identify clients' mobile phones in advance in order to determine whether they supported java, in order to avoid disappointing adolescent end-users.

Clinical testing of the mobile mood diary is ongoing and is being used as a means to refine the protocol for final use of the software. A nationwide clinic is currently evaluating the software. Several regional clinics and individual therapists are participating as well in order to provide evaluation results across different practices. While basic usability issues are not expected to be discovered at this stage of evaluation, past experience of clinical trials in the mental health area leads us to expect that issues related to the use of the content within sessions will emerge in the evaluation and feed back into subsequent versions of the diary.

\section{Discussion}

A significant advantage of the two first stages of evaluation was that the procedures, protocols and data analysis techniques were pinned down before clinical testing began. The school study raised a number of practical issues. For example, it became clear that it was important to develop fallback options in case the software did not work on a participant's phone which could result in them feeling left out. This is particularly important regarding mobile phones which have considerable social value for adolescents. During the initial installation of the software on phones, the software failed to work on the phones of two participants. Their reaction was that their phone was "not good enough". Experimenters reassured participants that whether the software worked on their phones had nothing to do with how 'good' their phones were. It is important to avoid this stigma. For clinical usage it may be useful to develop a simple tool which would allow therapists to enter the model of the client's phone to find out if the software is compatible with it.

It became clear, after the first two stages, that the manner in which the evaluation study was presented to both therapists and clients was critical. For example, when 
introducing the software to therapists interested in participating in trials, it was important to make it clear that we were not evaluating a system for two-way communication with clients. The school evaluation revealed trust issues relating to how the software was described to adolescents. At the outset, experimenters told participants they would be 'installing some software' on their mobile phones. This phrase made some adolescents suspicious of the testing, and potentially afraid they were being screened for mental health problems or that the software might "damage" their phones. Describing the installation as a "download" like a game or a ring tone rather than a program or an application was sufficient to allay such fears. The context and type of language used to describe new technologies to adolescents is important protocols which deal with these language and context issues would help experimenters and therapists when they are introducing new technologies to adolescents. In general the importance of how the software is introduced to all participants (e.g. to schools, parents, therapists, adolescent peers, adolescents) can not be overvalued.

Over the course of testing, privacy and security were concerns for many of the participants using paper diaries. Paper methods have drawbacks, such as being easy to lose or damage. They do not provide sufficient privacy to the end-user. Mobile phones can handle private information in a more unobtrusive fashion. The end-user needs to know exactly what the protocol for dealing with their information is, how much control they have over it and whether anyone else has access to it.

The school study provided good statistical data for the appeal and effectiveness of the mobile software. This was an important element in convincing therapists (often initially sceptical) to participate in evaluation. It also provides confidence in the likely outcomes at the clinical stage of evaluation.

The case study provides an illustration of the multi-stage prototyping methodology in action. This process provides the designer with a range of evaluation data that was not possible to obtain through other methods. Furthermore, it brought up significant difficulties with the software and protocols in safe situations before introducing a modified version into a sensitive situation. Usability, appeal and protocols were all tested in situations where there was time and the possibility of failure. This enabled the software design to be refined into one that could be used with confidence in this sensitive situation.

\section{CONCLUSION}

Mobile phone based software holds significant promise for engaging adolescents in therapeutic activities, but designing such applications is extremely challenging due to the ethical and access constraints which curtail access to end-users. This chapter has examined the design and evaluation approaches which may be used in these situations. We began by examining the characteristics and requirements that sets this area apart from other application areas, although relevant research from education and medical health care is considered. The emerging role of mobile technologies in therapeutic practice was explored, along with the identification of specific activities where it can have the most beneficial effect.

There are distinct barriers and limitations faced by researchers when designing software for mental health. These constraints render standard approaches to usercentred design impractical and potentially unethical. To address these issues, a set of 
design recommendations is presented. Designs should emphasize engagement, privacy and data security for the end-user. There is a need to work closely with therapists, and there are also benefits to developing systems that are easily adapted and modified by therapists. We have also discussed the typical measures available for data gathering for the evaluation of mobile applications and how suitable they are in this field.

We have presented a three stage prototyping process for the design and evaluation of mobile software for adolescents. The mobile mood diary case study provides an illustration of how the design recommendations, and particularly the multi-stage prototyping process, may operate in practice. Conducting initial evaluations with focus groups and then peer groups permits researchers to improve the software and to proceed to clinical evaluations with confidence in the outcomes.

Research on the design of technology within the area of mental health is still at an early stage. There is a need to establish which therapeutic tasks are best suited to mobility and how to design for them. At the moment, software is designed to compliment current therapeutic practice; in the future it may change practice to a degree. For the researcher, it can be difficult working in constrained and restricted circumstances, but positive results can have a dramatic impact on people's lives.

\section{REFERENCES}

Assay, T. P., \& Lambert, M. J. (1999). The Empirical Case for Common Factors in Therapy: Quantitative Findings. In B. L. Duncan, M. L. Hubble \& S. D. Miller (Eds.), The Heart and Soul of Change (pp. 23-55). Washington, DC: American Psychological Association.

Barendregt, W., \& Bekker, M. M. (2005). Extended guidelines for usability (and fun) testing with children, Proceedings of SIGCHI.nl Conference 2005, Den Haag.

Bauer, S., Percevic, R., Okon, E., Meerman, R., \& Kordy, H. (2003). Use of Text Messaging in the Aftercare of Patients with Bulimia Nervosa. European Eating Disorders Review, 11, 279-290.

BBC. (2006). Youngsters reliant on mobiles, retrieved 5/3/2007, from http://news.bbc.co.uk/2/hi/business/5358784.stm

BMA. (2006). Child and adolescent mental health - a guide for healthcare professionals (British Library No. ISBN: 1-905545-09-6). London: Board of Science of the British Medical Association.

Branch, J. L. (2001). Junior high students and Think Alouds Generating information-seeking process data using concurrent verbal protocols. Library \& Information Science Research, 23, 107-122.

Brukman, A., \& Bandlow, A. (2002). HCI for Kids. In J. J. a. A. Sears. (Ed.), Handbook of HumanComputer Interaction NJ: Lawrence Erlbaum Associates.

Carroll, J., Howard, S., Peck, J., \& Murphy, J. (2002). A Field Study of Perceptions and Use of Mobile Telephones by 16 to 22 year olds. Journal of Information Technology Theory and Application.

Caspar, F. (2004). Technological Developments and Applications in Clinical Psychology: Introduction. Journal of Clinical Psychiatry, 60(3), 221-238.

Coyle, D., Doherty, G., Matthews, M., \& Sharry, J. (2007). Computers in Talk-Based Mental Health Interventions. Interacting with Computers, In Press, DOI: 10.1016/j.intcom.2007.02.001.

Coyle, D., Matthews, M., Sharry, J., Nisbet, A., \& Doherty, G. (2005). Personal Investigator: A Therapeutic 3D Game for Adolescent Psychotherapy. International Journal of Interactive Technology and Smart Education, 2, 73-88.

Druin, A. (2002). The role of children in the design of new technology. Behaviour and Information Technology, 21, 1-25. 
Egan, G. (2002). The Skilled Helper: A Problem-Management and Opportunity-Development Approach to Helping (7th ed.). Pacific Grove, CA, US: Wadsworth Publishing.

Feldman Barrett, L., \& Barrett, D. J. (2001). An introduction to computerized experience sampling in psychology. Soc. Sci. Comput. Rev., 19, 175-185.

Gosbee, J., \& Ritchie, E. (1997). Human-computer interaction and medical software development. interactions, 4(4), 13-18.

Grinter, R. E., \& Eldridge, M. (2001). Y do tngrs lv 2 txt msg? Why "texting" became popular with teenagers. . Paper presented at the Proceedings of the Seventh European Conference on Computer Supported Cooperative Work (ECSCW)September 18-20 Bonn, Germany.

Hanna, L., Risden, K., \& Alexander, K. J. (1997). Guidelines for usability testing with children. . Interactions, 4 (5), 9-14.

Hanna, L., Risden, K., Czerwinski, M., \& Alexander, K. (1999). The role of usability research in designing children's computer products. . In A. Druin (Ed.), The Design of Children's Technology (pp. 3-26). San Francisco, CA: Morgan Kaufman.

Lester, J. S., B. \& Stelling, G. (1999). Lifelike Pedagogical Agents for Mixed-initiative Problem Solving in Constructivist Learning Environments. User Modeling and User-Adapted Interaction, 9(12), 1-44.

Leysia, P., \& Marilyn, S. (2002). Voice-mail diary studies for naturalistic data capture under mobile conditions, Proceedings of the 2002 ACM conference on Computer supported cooperative work. New Orleans, Louisiana, USA: ACM Press.

Markopoulos, P., \& Bekker, M. (2003). On the assessment of usability testing methods for children. Interacting with Computers, 15(2), 227-243.

Murray, C. L., \& Lopez, A. D. (Eds.). (1996). The Global Burden of Disease: A comprehensive assessment of mortality and disability from disease, injuries, and risk factors in 1990 and projected to 2020. Cambridge, MA: Harvard University.

Naismith, L., Lonsdale, P., Vavoula, G., \& Sharples, M. (2004). Mobile Technologies and Learning(report commissioned by NESTA FutureLab). Birmingham: University of Birmingham.

Nakamoto, H., Kawamoto, A., Tanabe, Y., Nakagawa, Y., Nishida, E., Akiba, T., et al. (2003). Telemedicine system using a cellular telephone for continuous ambulatory peritoneal dialysis patients. Advances in Peritoneal Dialysis, 19, 124-129.

Neville, R., Greene, A., McLeod, J., Tracy, A., \& Surie, J. (2002). Mobile phone text messaging can help young people manage asthma. British Medical Journal., 325.

Norton, M., Wonderlich, S. A., Myers, T., Mitchell, J. E., \& Crosby, R. D. (2003). The use of palmtop computers in the treatment of bulimia nervosa. European Eating Disorders Review, 11(3), 231 - 242.

Offer, D., Howard, K. I., Schonert, K. A., \& Ostrov, E. (1991). To whom do adolescents turn for help? Differences between disturbed and nondisturbed adolescents. Journal of the American Academy of Child and Adolescent Psychiatry, 30(4), 623-630.

Office for National Statistics. (2005). Mental health in children and young people in Great Britain, 2004. London: HMSO.

Office of the Deputy Prime Minister. (2004). Mental health and social exclusion. Social Exclusion Unit report. London: ODPM.

Palmblad, M., \& Tiplady, B. (2004). Electronic diaries and questionnaires: Designing user interfaces that are easy for all patients to use. Quality of Life Research, 13, 1199-1207.

Roberts, L., \& Dyer, A. (Eds.). (2004 ). Concise Guide to Ethics in Mental Health Care. Washington, DC: American Psychiatric Publishing.

Roschelle, J., Sharples, M., \& Chan, T. W. (2005). Introduction to the special issue on wireless and mobile technologies in education. Journal of Computer Assisted Learning, 21(3), 159-161.

Royal College of Psychiatrists. (2004). Mental health and growing up (3e). Factsheets for parents, teachers and young people. . London: Gaskell. 
Sanderson, K., Andrews, G., Corry, J., \& Lapsley, H. (2003). Reducing the burden of affective disorders: is evidence-based health care affordable? Journal of Affective Disorders, 77(2), 109-125.

Savill-Smith, C. (2005). The use of palmtop computers for learning: a review of the literature. British Journal of Educational Technology, 36(3), 567-568.

Schaefer, C. E., \& Reid, S. E. (Eds.). (2001). Game play : therapeutic use of childhood games. New York: Chichester : Wiley.

Vavoula, G., Meek, J., Sharples, M., Lonsdale, P., \& Rudman, P. (2006). A Lifecycle approach to evaluating MyArtSpace. Paper presented at the Wireless, Mobile and Ubiquitous Technologies in Education (WMUTE) 2006.

Vavoula, G., Sharples, M., Rudman, P., Lonsdale, P., \& Meek, J. (2006). (in press). Learning Bridges: a role for mobile technologies in education. Educational Technology Magazine, special issue on mobile learning.

Wood, J., Keen, A., Basu, N., \& Robertshaw, S. (2003). The development of mobile applications for patient education. San Francisco, California: ACM Press 